\title{
State Space Reduction using Partial Order Techniques
}

\author{
E. M. Clarke ${ }^{1}$, O. Grumberg ${ }^{2 \star \star}$, M. Minea ${ }^{1}$, and D. Peled ${ }^{3}$ \\ 1 Carnegie Mellon University, School of Computer Science, Pittsburgh, PA 15213-3891, USA \\ 2 Dept. of Computer Science, The Technion, Haifa 32000, Israel \\ 3 Bell Laboratories, 600 Mountain Ave., Murray Hill, NJ 07974-2070, USA
}

August 17, 1998

\begin{abstract}
With the advancement of computer technology, highly concurrent systems are being developed. The verification of such systems is a challenging task, as their state space grows exponentially with the number of processes. Partial order reduction is an effective technique to address this problem. It relies on the observation that the effect of executing transitions concurrently is often independent of their ordering. In this paper we present the basic principles behind partial order reduction and its implementation.
\end{abstract}

\section{Introduction}

One of the main problems in automatic verification of systems is the so-called state space explosion problem. For many types of systems, the number of possible states during system execution grows exponentially with the size of the system and the number of its component parts. This quickly leads to models whose size exceeds the current capabilities of verification tools.

Partial order reduction is a technique that addresses this problem for concurrent asynchronous systems by constructing a smaller state space that is searched by the verification (model checking) algorithms. In general, asynchronous systems are described using an interleaving model of computation. Concurrent events are modeled by allowing their execution in all possible orders

\footnotetext{
* This research is sponsored by the the Semiconductor Research Corporation (SRC) under Contract No. 97-DJ-294, the National Science Foundation (NSF) under Grant No. CCR-9505472, and the Defense Advanced Research Projects Agency (DARPA) under Contract No. DABT63-96-C-0071. Any opinions, findings and conclusions or recommendations expressed in this material are those of the author(s) and do not necessarily reflect the views of SRC, NSF, DARPA, or the United States Government.

$\star \star$ This research was partially supported by the fund for the promotion of research at the Technion.

Correspondence to: Doron Peled
}

relative to each other, creating a large number of possible states and paths. However, specifications typically do not distinguish between all different orders. Partial order reduction considers only a restricted set of behaviors of the system, while guaranteeing that the ignored behaviors do not add any new information.

In this survey we will describe a method of partial order reduction. The main goal of this paper is to provide an intuitive description of the main ideas and present some techniques that can be used for implementation.

Reducing the state space by using commutativity between concurrent transitions was suggested by several researchers. In his Ph.D. thesis, Overman [20] suggested a method to avoid exploring all the states of a concurrent system. However, this method was only applied to systems without loops. Katz and Peled [16] suggested a proof system for concurrent systems that takes the commutativity between transitions into account. The core of the deduction system was based on using proof rules that asserted properties of sequences which are generated by taking certain subsets of successors from each state.

In the last decade, several researchers have developed methods to apply reduction principles in model checking. These techniques include the stubborn sets method of Valmari [24], the persistent sets method of Godefroid and Wolper [12, 11], and the ample sets method of Peled [22]. These works contain similar ideas, although they differ with respect to the details of the suggested reduction. We will present here the ample sets method.

The name partial order reduction reflects a connection between the initial versions of these reductions and partial order semantics. Roughly, a partially ordered execution is represented by a set of events and a causality relation between them. The causality relation indicates that some events must precede others, while events that are not constrained by this relation are independent and can happen in any order. In contrast, in a total ordering on events, any given event must either precede or follow 
any other event. Some versions of partial order reduction guarantee that the reduced state space includes for each such partially ordered execution at least one linearization (completion into a total order). However, most current methods do not maintain this relation anymore.

\section{Fundamental Notions}

The systems that we analyze are modeled as state transition graphs. If $S$ is the set of states, a transition is a relation $\alpha \subseteq S \times S$, i.e., it can be taken between different pairs of states. A state transition graph is then defined as a tuple $M=\left(S, S_{0}, T, L\right)$, where $S_{0} \subseteq S$ is a set of initial states, $T$ is a set of transitions $\alpha \subseteq S \times S$, and $L: S \rightarrow 2^{A P}$ is a labeling function that assigns to each state a subset of some set $A P$ of atomic propositions.

A transition $\alpha \in T$ is enabled in a state $s$ if there exists a state $s^{\prime}$ such that $\left(s, s^{\prime}\right) \in \alpha$ (or in other words $\alpha\left(s, s^{\prime}\right)$ holds). If for any state $s$ there is at most one state such that $\alpha\left(s, s^{\prime}\right)$, we call $\alpha$ a deterministic transition. In this case we can view $\alpha$ as a partial function on states instead of a relation and write $s^{\prime}=\alpha(s)$ instead of $\alpha\left(s, s^{\prime}\right)$. The following presentation considers only deterministic transitions, without further explicit mention.

We reason about execution sequences of the system, called paths. A path in a state-transition graph $M$ is a finite or infinite sequence $\sigma=s_{0} \stackrel{\alpha_{\rho}}{\rightarrow} s_{1} \stackrel{\alpha_{1}}{\rightarrow} \ldots$ such that $s_{i+1}=\alpha_{i}\left(s_{i}\right)$ for every $i$.

In asynchronous systems, the number of transitions occurring between two events has no direct relationship to the time delay between them. Furthermore, transitions which are concurrent in the system appear serialized in some order in the interleaving model. These observations argue for a specification which cannot distinguish between sequences of identically labeled states on an execution path of the system.

We call two infinite paths stuttering equivalent if they have identical state labelings after in each of them, any finite sequence of identically labeled states is collapsed to a single state. In other words, two infinite paths $\sigma=$ $s_{0} \stackrel{\alpha_{\rho}}{\rightarrow} s_{1} \stackrel{\alpha_{1}}{\rightarrow} \ldots$ and $\rho=r_{0} \stackrel{\beta_{0}}{\rightarrow} r_{1} \stackrel{\beta_{1}}{\rightarrow} \ldots$ are stuttering equivalent if one can define two infinite sequences of integers $0=i_{0}<i_{1}<\ldots$ and $0=j_{0}<j_{1}<\ldots$ such that $\forall k \geq 0, L\left(s_{i_{k}}\right)=L\left(s_{i_{k}+1}\right)=\ldots=L\left(s_{i_{k+1}-1}\right)=$ $L\left(r_{j_{k}}\right)=L\left(r_{j_{k}+1}\right)=\ldots=L\left(r_{j_{k+1}-1}\right)$. The indices $i_{k}$ and $j_{k}$ are the starting points of identically labeled subsequences of states in the two paths, respectively. The stuttering equivalence relation between $\sigma$ and $\rho$ is denoted by $\sigma \sim_{s t} \rho$.

The temporal logic LTL [8] allows assertions about the temporal behavior of a program. Given a finite set of propositions $A P$, the LTL formulas are defined inductively as follows:

- every member of $A P$ is a formula,

- if $\varphi$ and $\psi$ are formulas, then so are $\neg \varphi, \varphi \wedge \psi, \bigcirc \varphi$ and $\varphi \mathcal{U} \psi$.

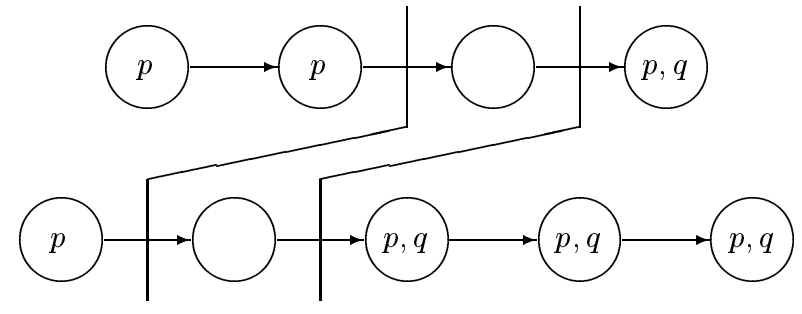

Fig. 1. Stuttering equivalent paths

An interpretation of an LTL formula is an infinite word $\xi=x_{0} x_{1} \cdots$ over the alphabet $2^{A P}$, i.e. a mapping from the naturals to $2^{A P}$. We write $\xi_{i}$ for the suffix of $\xi$ starting at $x_{i}$. The semantics of LTL is as follows:

$-\xi \models p$ iff $p \in x_{0}$, for $p \in A P$,

$-\xi \models \neg \varphi$ iff not $\xi=\varphi$,

$-\xi \models \varphi \wedge \psi$ iff $\xi \models \varphi$ and $\xi \mid \psi$,

$-\xi \models \bigcirc \varphi$ iff $\xi_{1}=\varphi$,

$-\xi=\varphi \mathcal{U} \psi$ iff there is an $i \geq 0$ such that $\xi_{i} \models \psi$ and

$\xi_{j}=\varphi$ for all $0 \leq j<i$.

Let false as an abbreviation for $A \wedge \neg A$, and true be an abbreviation for $\neg$ false. We also use the following abbreviations: $\varphi \vee \psi=\neg((\neg \varphi) \wedge(\neg \psi)), \diamond \varphi=\operatorname{true} \mathcal{U} \varphi$, $\square \varphi=\neg \diamond \neg \varphi$.

Given a state transition graph $M$ and an LTL formula $\varphi$, the model checking problem for $M$ and $\varphi$ is to verify that for every initial state $s_{0} \in S_{0}$ and every path $\xi$ starting in $s_{0}$, it is true that $\xi=\varphi$. If this holds, we write $M \models \varphi$.

An LTL formula $\varphi$ is invariant under stuttering if for any two paths $\sigma$ and $\sigma^{\prime}$ such that $\sigma \sim_{s t} \sigma^{\prime}$, we have $\sigma \mid=\varphi$ iff $\sigma^{\prime} \mid=\varphi$.

In general, an LTL formula can be sensitive to stuttering if it contains the next-time operator $\bigcirc$. Denote by LTL $_{-X}$ the subset of logic LTL that does not make use of the next-time operator. Peled and Wilke show [23] that an LTL property is invariant under stuttering iff it can be expressed in LTL $_{-X}$.

The notion of stuttering equivalence can be extended from paths to state transition graphs. Two state transition graphs $M$ and $M^{\prime}$ are stuttering equivalent iff the following two symmetric conditions hold:

- for each path $\sigma$ from an initial state of $M$ there is a path $\sigma^{\prime}$ from an initial state of $M^{\prime}$ such that $\sigma \sim_{s t} \sigma^{\prime}$. - for each path $\sigma^{\prime}$ from an initial state of $M^{\prime}$ there is a path $\sigma$ from an initial state of $M$ such that $\sigma^{\prime} \sim_{s t} \sigma$.

From the definition of stuttering equivalence of state transition graphs and the theorem about stuttering invariance of $\mathrm{LTL}_{-X}$ formulas, one can deduce the following result:

If $M$ and $M^{\prime}$ are state transition graphs which are stuttering equivalent, then for any $\mathrm{LTL}_{-X}$ property $\varphi, M \mid=\varphi$ iff $M^{\prime}=\varphi$. 
This result justifies the use of partial order reduction by virtue of the fact that it produces a structure that is stuttering equivalent to the original state transition graph.

\section{Principles of Partial Order Reduction}

As mentioned in the introduction, one of the main reasons for state space explosion in asynchronous systems is that the interleaving model of computation must consider all possible event orderings, in order to avoid the omission of any particular one. However, since interleaving is introduced to model concurrency, for independent transitions this ordering is often irrelevant. On the other hand, depending on the specification language, it is possible that the property to be verified is actually able to discriminate between behaviors that only differ by this ordering. To be able to use partial order reduction, it is necessary to have a specification that does not distinguish between such behaviors and a procedure that selects a set of behaviors that constitutes the reduced model. If some behavior is not present in the reduced model, an equivalent one has to be included in order to guarantee correctness.

To illustrate the importance of reduction, consider a system composed of $n$ concurrent processes, $P_{1}$ through $P_{n}$. Each process $P_{i}$ has a transition $\alpha_{i}$ enabled in some local state $s_{i}$, that changes the value of the labeling function: $\alpha_{i}\left(s_{i}\right)=s_{i}^{\prime}, L\left(s_{i}\right)=\emptyset, L\left(s_{i}^{\prime}\right)=\{p\}$, for some $p \in A P$. The concurrent transitions $\alpha_{i}$ can be ordered in $n$ ! possible ways, producing a total of $2^{n}$ different states. Yet it is possible that the specification only needs to establish a property that links the initial global state $\left(s_{1}, \ldots, s_{n}\right)$ with the resulting state $\left(s_{1}^{\prime}, \ldots, s_{n}^{\prime}\right)$, irrespective of the path taken between these. In this case, it is much more efficient to consider only one particular ordering and the corresponding $n+1$ states.

Typically, the reduced model is constructed by performing a modified depth-first search on an explicit state representation of the system. Model checking is done in a separate phase, on the resulting reduced state transition graph. It is also possible to construct the reduced model on the fly, while performing model checking. Other variations are to use breadth-first search instead of depth-first search, or to combine partial order reduction with symbolic model checking. A common point for all variants is that the reduced state space is constructed directly, without ever building the full state graph. This would be counter to the purpose of reduction, since it is likely that the full state graph is too large to be constructed in the first place.

Consider, for the purpose of illustration, the case of depth-first search. A typical search that constructs the entire reachable state space would follow all transitions enabled at the current state in the search. With partial order reduction, only a subset of the enabled transitions is expanded at each state $s$. We will call this set ample $(s)$.
To apply this method, we need a procedure to compute a suitable set $\operatorname{ample}(s)$ for every state $s$. First, in order to obtain a much smaller state graph, ample $(s)$ has to be significantly smaller than enabled $(s)$. On the other hand, to ensure the correctness of the reduction, ample $(s)$ has to include enough transitions such that for each behavior in the full state graph there is an equivalent behavior in the reduced state graph. Finally, computing an ample set should be done with a reasonably small overhead so that verification time is not increased compared to full state space search.

Since the key issue in partial order reduction is to select only a restricted number of orderings between transitions for analysis, the concept of transitions that can be reordered has to be formalized. This can be done by defining the key concept of independence relation between transitions. Two transitions $\alpha, \beta \in T$ are independent if they satisfy the following two conditions for each state $s \in S$ :

Enabledness:

If $\alpha, \beta \in$ enabled $(s)$ then $\alpha \in \operatorname{enabled}(\beta(s))$ and $\beta \in \operatorname{enabled}(\alpha(s))$.

\section{Commutativity:}

If $\alpha, \beta \in \operatorname{enabled}(s)$ then $\alpha(\beta(s))=\beta(\alpha(s))$.

The enabledness condition expresses the fact that two independent transitions that are enabled at a given state cannot disable each other. Note that the definition given here allows independent transitions to enable one another. The commutativity condition states that the execution of two independent transitions in any order (which is guaranteed to be possible by the enabledness condition) leads to the same state. Two transitions are called dependent if they are not independent.

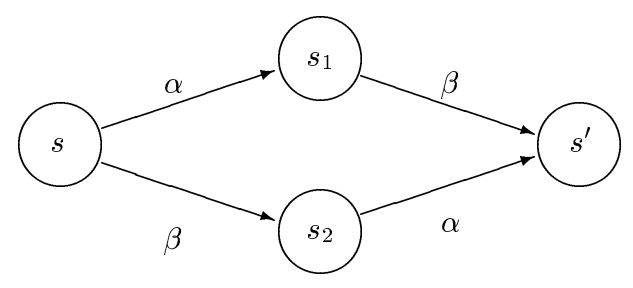

Fig. 2. Independent transitions

Consider the simple fragment of a state transition graph depicted in Figure 2. If transitions $\alpha$ and $\beta$ are independent, a possible reduction would be consider only the execution sequence $s \stackrel{\alpha}{\rightarrow} s_{1} \stackrel{\beta}{\rightarrow} s^{\prime}$ and not the path $s \stackrel{\beta}{\rightarrow} s_{2} \stackrel{\alpha}{\rightarrow} s^{\prime}$. However, this reduction may not be necessarily correct, either because the checked property can distinguish between the intermediate states $s_{1}$ and $s_{2}$, or because eliminating one of these states may cause some of its successors (which are significant for verification) not to be explored. Additional conditions for the correctness of the reduction are needed, and they will be described in the following. 
To address the first of these two issues, we define what it means for a specification to distinguish between two states, by introducing a second key concept, of invisible transitions. Recall that $L: S \rightarrow 2^{A P}$ is the labeling function that assigns to each state a set of atomic propositions. The specification does not necessarily refer to the entire set of atomic propositions; let $A P^{\prime} \subseteq A P$ be the subset of atomic propositions referenced in the specification. We call a transition $\alpha$ invisible with respect to some subset $A P^{\prime} \subseteq A P$ if its execution between any two states does not change the labeling with atomic propositions from $A P^{\prime}$. Formally, the transition $\alpha \in T$ is invisible with respect to $A P^{\prime}$ if for any two states $s, s^{\prime} \in S$ such that $s^{\prime}=\alpha(s)$ we have $L(s) \cap A P^{\prime}=L\left(s^{\prime}\right) \cap A P^{\prime}$. A transition is visible if it is not invisible. If the subset of atomic propositions $A P^{\prime}$ is clear from the context (it is usually the set of atomic propositions contained in the specification), we will simply say that a transition is visible or invisible without explicitly mentioning that this is with respect to $A P^{\prime}$.

\section{Partial Order Reduction for LTL $_{-X}$}

We have seen in the previous section that the properties of independence and invisibility for transitions and stuttering invariance for $\mathrm{LTL}_{-X}$ formulas allow us to verify the specification for the given system on a reduced model, and thus avoid the generation of all states. The reduced model is constructed by selecting at each step a subset ample $(s)$ of the transitions which are enabled at the current state $s$. We say that a node $s$ is fully expanded if ample $(s)=\operatorname{enabled}(s)$.

We need a procedure that will determine a suitable set of ample transitions at each state. Rather than directly give an algorithm that solves this problem, in this section we will characterize the set ample(s) using a set of conditions. The next section continues by describing various heuristics that can be used to find ample sets that satisfy these conditions.

The first condition is trivial and guarantees that the search algorithm with reduction will make progress if the normal search algorithm would:

C0 Emptiness ample $(s)=\emptyset$ iff enabled $(s)=\emptyset$.

The next constraint is introduced to ensure that any path that is not included in the reduced state-transition graph can be transformed, based on the properties of independent transitions, into a path in the reduced model, and therefore the reduction does not omit any paths which are essential for verification.

C1 Ample decomposition In the full state graph, on any path starting from some state $s$, a transition dependent on a transition from ample (s) cannot appear before some transition from ample(s) is executed.

To analyze the implications of $\mathbf{C 1}$, consider an arbitrary sequence of transitions $\sigma=\alpha_{0}, \alpha_{1} \ldots$ that can be taken from some state $s_{0}$ in the full state transition graph. We outline the basic ideas of a construction that can be used to generate a path in the reduced model that contains all transitions from $\sigma$. More details of the construction and a proof for its correctness are given by Clarke, Grumberg and Peled [4].

(a) if $\alpha_{0} \in$ ample $\left(s_{0}\right)$, then $\alpha_{0}$ can be taken from $s_{0}$ in the reduced model, and the path prefix $s_{0} \stackrel{\alpha_{0}}{\rightarrow} s_{1}$ belongs to the reduced model. The construction is continued inductively from $s_{1}$.

(b) if $\alpha_{0} \notin$ ample $\left(s_{0}\right)$, consider first the case where the transition sequence $\sigma$ contains some transition from ample $\left(s_{0}\right)$. Let $\beta$ be the first such transition appearing in $\sigma$, i.e. $\beta=\alpha_{k}$, with $k \geq 1$. Then by condition $\mathbf{C 1}$ all transitions $\alpha_{i}$ with $0 \leq i<k$ must be independent of $\beta$, and thus commute with it. Therefore the transition sequence $\beta \alpha_{0} \alpha_{1} \ldots \alpha_{k-1} \alpha_{k+1} \ldots$ is also a transition sequence enabled in $s_{0}$ in the original model. Moreover, since $\beta \in \operatorname{ample}\left(s_{0}\right)$, the first transition can also be taken in the reduced model and the construction continues form $s_{1}=\beta\left(s_{0}\right)$.

(c) if $\alpha_{0} \notin$ ample $\left(s_{0}\right)$ and the sequence $\sigma$ does not contain any transition from ample $\left(s_{0}\right)$, let $\beta$ be an arbitrary transition from ample $\left(s_{0}\right)$. By condition $\mathbf{C 1}$, none of the transitions in $\sigma$ can be dependent on $\beta$. Therefore, if $s_{1}=\beta\left(s_{0}\right)$, the path $s_{0} \stackrel{\beta}{\rightarrow} s_{1}$ belongs to the reduced model and the transition sequence $\sigma$ is executable after $s_{1}$.

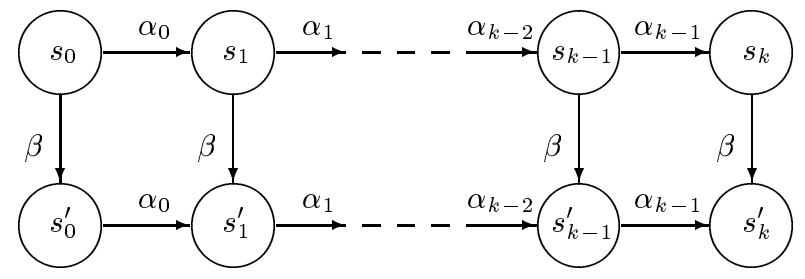

Fig. 3. Reordering of transitions based on commutativity

Transforming a path $\sigma$ in the full state transition graph into a path $\sigma^{\prime}$ including all transitions from $\sigma$ and with a prefix that belongs to the reduced model is not sufficient. We still need to know that the constructed path is stuttering equivalent to the initial one, so that the truth value of the specification will not be affected.

C2 Invisibility If a state $s$ is not fully expanded, every transition $\alpha \in$ ample $(s)$ has to be invisible.

To analyze the effect of this condition, consider cases (b) and (c) discussed previously in conjunction with C1 (there is no need to discuss (a), since it did not imply any change to the path). For case (b), denote the $i^{\text {th }}$ edge on $\sigma$ by $s_{i} \stackrel{\alpha_{i}}{\rightarrow} s_{i+1}$, and let $s_{i}^{\prime}=\beta\left(s_{i}\right)$. Since $\beta$ commutes with $\alpha_{i}$ for $i<k$, it follows that $s_{0}^{\prime} s_{1}^{\prime} \ldots s_{k-1}^{\prime} s_{k+1}$ is exactly the state sequence obtained by executing the transition sequence $\beta \alpha_{0} \ldots \alpha_{k-1}$. Since $\beta \in \operatorname{ample}\left(s_{0}\right)$ but $\alpha_{0} \notin \operatorname{ample}\left(s_{0}\right)$, it follows by $\mathbf{C 2}$ that $s_{0}$ is not 
fully expanded, therefore $\beta$ has to be invisible. Consequently, $L\left(s_{i}^{\prime}\right)=L\left(s_{i}\right) \forall i \leq k$. and therefore the sequences $s_{0} s_{1} \ldots s_{k} s_{k+1} \ldots$ and $s_{0} s_{0}^{\prime} s_{1}^{\prime} \ldots s_{k-1}^{\prime} s_{k+1} \ldots$ are stuttering equivalent. Case (c) is similar: Here too, $\beta$ has to be invisible and $L\left(\beta\left(s_{i}\right)\right)=L\left(s_{i}\right)$ for any $i$, therefore the two state sequences are stuttering equivalent.

Together, C1 and C2 still do not guarantee that a stuttering equivalent path in the reduced model can be found for any path in the original model. To see this, we note that the recursive condition we have outlined is not guaranteed to produce a path that contains all transitions in the original path $\sigma$. For case (c), none of the original transitions in $\sigma$ is "consumed". Instead, an auxiliary transition $\beta \in$ ample $\left(s_{0}\right)$ is appended to the beginning of $\sigma$. If $\sigma$ does not contain any ample transition from $\beta\left(s_{0}\right)$ either and this step is repeated sufficiently often, a cycle consisting of inserted ample transitions will be closed. Therefore, the expansion of state $s_{0}$ will terminate without ever considering the transition $\alpha_{0}$ which is enabled in that state. If $\alpha_{0}$ is a visible transition, the specification may have different truth values in the original and reduced models. To avoid this case, it is necessary to introduce a third condition:

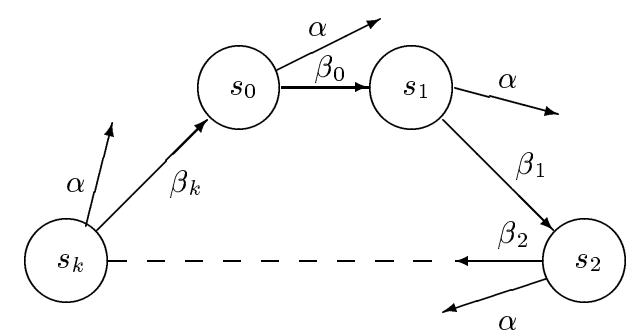

Fig. 4. Cycle-closing condition

C3 Cycle closing condition If a cycle contains a state in which some transition $\alpha$ is enabled, then it also contains a state $s$ such that $\alpha \in$ ample $(s)$.

With C3, the set of conditions that have to be satisfied by ample sets is complete. The next section shows how sets of transitions satisfying these conditions can be computed in practice.

\section{Calculating Ample Sets}

To obtain an efficient reduction procedure, it is necessary to determine values for the ample sets at each state that result in a significantly smaller number of successor states and are at the same time easy to compute with small overhead. We describe in the following how this can be done for each of the given conditions.

Checking that an ample set is nonempty (condition C0) is trivial. Likewise, to verify condition $\mathbf{C 2}$ it suffices to examine each transition in the ample set of a state. Condition $\mathbf{C 1}$ however, is more difficult. One reason is that $\mathbf{C 1}$ is stated as a property of the full state transition graph, which the reduction technique attempts to avoid in the first place. Second, the formulation of $\mathbf{C 1}$ refers to future states that may not have been yet examined in the search. It can be shown [4] that in general checking C1 is at least as hard as checking reachability for the full state transition graph.

Consequently, rather than using an algorithm that needs to check $\mathbf{C 1}$ for an arbitrary set of transitions, in general we will exploit the structure of the system to produce sets of ample transitions for which condition C1 is guaranteed to hold. The following exposition discusses such algorithms for two different classes of concurrent systems. Common to both is the modeling of the system as a set of processes, each with a set of transitions (that may be common in several processes). A process has a set of local variables that can be changed only by transitions performed by that process. These variables are part of the local state of the process, and the product of the local states forms the global state of the system. A transition that only changes the local variables of a process is called an internal transition.

The synchronous communication model requires the sender and the receiver to coordinate, such as for instance in Communicating Sequential Processes [13] or the rendezvous model of ADA. Since the sending and the receiving transitions happen simultaneously, they can be considered as a common transition shared by the two processes. We call such a transition a communication transition. Assume for the following that a system has only local and synchronous communication transitions. When a process $P_{i}$ arrives at a communication point (a send or receive action), the corresponding communication transition is enabled by $P_{i}$. It will only be enabled globally when the communication partner of $P_{i}$ arrives at its corresponding transition point. A communication transition between two processes $P_{i}$ and $P_{j}$ is said to be locally enabled by $P_{i}$ at state $s$ if it can be executed from some state $s^{\prime}$ that has the same local state of $P_{i}$ as $s$.

A conservative definition of the dependence relation considers all local transitions within a process to be pairwise dependent. Therefore, a communication transition will be dependent on local transitions from both processes. A suitable selection of ample sets that will satisfy C1 is the following:

For a state $s$, select a subset $\mathcal{P}$ of processes, such that for any $P_{i} \in \mathcal{P}$ there is no communication locally enabled by $P_{i}$ with a process outside of $P$. Then select all transitions enabled in state $s$ and belonging to some process in $\mathcal{P}$ as the set ample $(s)$.

The partitioning of the processes in two sets guarantees that by executing transitions outside the ample set it is not possible that a transition dependent on an ample transition will become globally enabled and therefore executed before a transition in the ample set. This is exactly the constraint imposed by $\mathbf{C 1}$. The new rule can be applied in practice by initially selecting $\mathcal{P}$ to consist of a single process. If the constraint is not satisfied, other processes are added to $\mathcal{P}$ until it holds. In the worst case, 
$\mathcal{P}$ is the set of all processes, which corresponds to a fully expanded state and no reduction at all.

In the asynchronous communication model, in addition to the local variables of each process, there are shared message queues through which the communication between processes is performed. A process executing a send operation does not have to wait for the destination process to execute the appropriate receive, unless the message queue that would be used for communication is full (we assume a finite-state system and therefore finite queues). Likewise, a receiving process consumes a message from its input queue and does not block unless this queue is empty. We call a send and a receive operation matching if they share the same message queue. For this discussion we assume the existence of a message queue for each pair of communicating processes; different rules can be given for other cases (e.g. one input queue per process).

Note that a send operation can enable a matching receive (if the queue was previously empty) and conversely, a receive operation can enable a matching send (if the queue was previously full). Both of these scenarios are allowed for the definition of transition independence, which merely requires that transitions do not disable each other. Consequently, we consider the following constraint:

For a given state $s$, select as the set ample $(s)$ the set of all enabled transitions from a set of processes $\mathcal{P}$ satisfying the following two conditions:

- No send transition of a process in $\mathcal{P}$ is blocked only because a process outside of $\mathcal{P}$ has the corresponding queue full.

- No receive transition of a process in $\mathcal{P}$ is blocked only because a process outside of $\mathcal{P}$ has the corresponding queue empty.

The above first condition guarantees that the following scenario does not happen:

A sequence of transitions from processes outside of $\mathcal{P}$ is executed from the current state. Among these transitions, which are independent of those selected, there is eventually a receive transition $\gamma$. Its execution enables a send transition $\alpha$ of some process in $\mathcal{P}$, as its queue is no longer full. But $\alpha$ is dependent on some transition in the selected ample set, contradicting $\mathbf{C 1}$.

With the first condition above, such a transition $\gamma$ cannot exist. A similar scenario that justifies the second condition can also be shown.

As in the previous case, we can implement this rule by starting with $\mathcal{P}$ consisting of an arbitrary process $P_{i}$ and selectively adding other processes until the communication conditions are satisfied.

The original formulation of $\mathbf{C} \mathbf{1}$ requires knowledge about transitions that can be executed in the future. Additional information gathered through preliminary static analysis may allow more flexibility in choosing ample sets. For instance, the condition given above for the case of synchronous communication can be weakened. A process from $\mathcal{P}$ is allowed to have a locally enabled communication transition with a process outside $\mathcal{P}$ if one can determine that this communication cannot actually take place in any state reachable from the current state.

However, checking that from a given state a transition is disabled in the future is as hard as the model checking problem itself. Again, the solution is to use an analysis that will identify some of the transitions that can no longer become enabled starting from the current state, rather than all of them. This can be done by performing a separate reachability analysis for each process and taking advantage of the fact that the state space of a single process is much smaller than the global state space. In the example given above for synchronous communication one could check whether the matching communication transition can be reached in the other process starting from its local state. This analysis assumes that all communication transitions which are joint with other processes are enabled by those processes, and is therefore conservative. Moreover, it is also possible to ignore data values (selectively or completely) and perform in the simplest case only a static analysis of the control flow graph of the process.

This search can be done in a preliminary stage of the reduction algorithm. It can identify unreachable transitions among those transitions that have possible dependencies (synchronous/asynchronous communication, use of global variables, etc.). During the subsequent state search, this information can be used to identify more subsets as ample sets and thus improve the efficiency of the reduction.

\section{Experience with Partial Order Reduction}

Various systems that use partial order reduction algorithms have been implemented. Our experience is mainly related to the SPIN implementation described by Holzmann and Peled [15]. In this section we will describe various lessons learned about the difficulty of implementation and the efficiency of the partial order reduction.

One noticeable fact about partial order reduction is that it is usually given as a set of principles rather than an algorithm, which calls for an open-ended list of heuristics. In particular, condition $\mathbf{C 1}$ can be satisfied by a trivial implementation that selects all the enabled transitions from any given state. A better implementation performs an analysis which is based e.g., on the types of transitions that are enabled or disabled from the current state. By making the analysis more involved, and taking more cases into consideration, one can obtain a better reduction. On the other hand, the overhead may also grow when using a more complicated analysis.

In the SPIN implementation [15], the initial decision was to provide an adequate reduction for the asynchronous communication case. SPIN uses the strategy 
for calculating ample sets presented in Section 5. In particular, Spin looks for a singleton set $\mathcal{P}$, i.e., one process that satisfies these conditions. When systems that use other concurrency mechanisms such as shared variables or synchronous communication are verified, the reduction might be far from optimal. Even with this restriction, the size of the SPIN code roughly doubled when the partial order reduction was first added to it.

The effectiveness of the reduction obtained using the partial order techniques varies considerably among different examples. It is immediately observable that the method is effective only for asynchronous systems, where commutativity between concurrent transitions can be exploited. Thus, for most hardware systems, which are usually synchronous, there is little, if any reduction. Concurrent systems, and in particular distributed programs, which exhibit a lot of parallelism and independency, are the main focus of the partial order reduction. For certain examples, e.g., the distributed sieve of Eratosthenes for calculating prime numbers, a version of concurrent sorting and a leader election in a ring of processes, the reduction was shown to improve exponentially with the number of processes. Some other, more typical examples show reduction by one order of magnitude.

A factor that greatly influences the effectiveness of the reduction is based on the following observation: when two transitions that are independent can both change the truth value of predicates that appear in the checked property (i.e., the transitions are visible), the order between them becomes relevant, even if they may be independent. Specifically, Condition C2 forces visible transitions to be selected into an ample set only with all other visible transitions. A closer look shows that as a result, if an execution is not present because of the reduction, another execution with the same order of visible transitions will be present in the reduced state graph. Experimentally, the effectiveness of the reduction diminishes quickly with the number of predicates used in the specification. For this reason, it is useful to try to simplify the checked properties, e.g., checking separately for $\diamond p$ and for $\diamond q$, rather than $\diamond p \wedge \diamond q$.

In Table 1, we present some experimental results of using partial order reduction. The experiments where performed on a SGI Challenge machine with 12 processors and 1.28 Gigabytes of memory. The checked algorithms were as follows:

sieve The distributed Sieve of Eratosthenes algorithm for finding prime numbers.

$d t p \quad$ A data transfer protocol.

snoopy A cache coherence protocol.

pftp A file transfer protocol.

These examples are included in the standard benchmark that is distributed with the SPIN model checking system [14]. The SPIN system, including its standard example protocols, can be obtained from the web page http://netlib.bell-labs.com/netlib/spin/whatispin.html.

\begin{tabular}{|lr|r|r|r|r|}
\hline $\begin{array}{l}\text { Algo- } \\
\text { rithm }\end{array}$ & $\begin{array}{r}\text { Reduc- } \\
\text { tion }\end{array}$ & States & $\begin{array}{r}\text { Transi- } \\
\text { tions }\end{array}$ & Memory & Time \\
\hline sieve & No & 10,878 & 35,594 & 2,315 & 1.68 \\
& Yes & 157 & 157 & 1,078 & 0.08 \\
\hline dtp & No & 251,409 & 648,467 & 34,540 & 32.2 \\
& Yes & 16,459 & 17,603 & 3,582 & 1.47 \\
\hline snoopy & No & 164,258 & 546,805 & 19,979 & 33.57 \\
& Yes & 29,796 & 44,145 & 4,614 & 3.58 \\
\hline pftp & No & 514,188 & $1,138,750$ & 70,004 & 123.34 \\
& Yes & 125,595 & 191,466 & 18,057 & 18.59 \\
\hline
\end{tabular}

Table 1. Experimental results for partial order reduction

For each of these algorithms, the property that was checked asserted that some variable, initialized with 0 , eventually becomes 1 .

The Sieve of Eratosthenes shows the best reduction among the four protocol listed above. Furthermore, when checking this protocol with a growing number of processes, one can measure an exponential blowup in the the number of states when the reduction is not applied, and a linear growth with the reduction. This demonstrates an exponential reduction. The reason for this is that all this protocol's executions are essentially equivalent up to reordering of independently executed transitions, as there are no nondeterministic choices in the code. The exponential explosion in the state space follows entirely from different arrangement of these transitions. In the other checked protocols, a more typical reduction is achieved, as they exhibit both concurrency and nondeterminism.

\section{Other Partial Order Reduction Methods}

The ample sets algorithm, and similarly the persistent sets and stubborn sets algorithms are based on calculating a subset of successors that generates enough paths to preserve the checked property. This is done by analyzing the current state and the enabled and disabled transitions of the checked system.

A different reduction principle has been suggested by Godefroid [10]. The sleep set method, originally developed for detecting deadlocks, generates a reduced state graph by observing the transitions that were already explored. For each node $s$ expanded by the algorithm, a set of transitions sleep $(s)$ is kept. This is the set of transitions that one does not need to explore from $s$. The intuition behind the sleep sets algorithm is as follows: If a transition $\alpha$ is already explored from some node $s$, then when any transition $s \stackrel{\beta}{\rightarrow} t$, with $\beta$ independent of $\alpha$ is explored, there is no need to explore the transition $\alpha$ from $t$ and $\alpha$ is added to $\operatorname{sleep}(t)$. This follows from the fact that when the expansion of $\alpha$ is finished, enough representatives for transitions following the execution of $\alpha$ (including $\beta$ ) are explored, and exploring $\alpha$ after $\beta$ would lead to the same state as exploring $\beta$ after $\alpha$. Moreover, consider the case when $\alpha$ need not be 
explored from some node $s$ (i.e., $\alpha \in \operatorname{sleep}(s)$ ) and an edge $s \stackrel{\gamma}{\rightarrow} r$ with $\gamma$ independent of $\alpha$ is explored. Then $\alpha$ is added to sleep $(r)$, since the occurrences of $\alpha$ immediately following $\gamma$ can be commuted to represent an already unnecessary sequence.

When a node is reached again during expansion, a new sleep set is calculated for it, and is compared with the one it had before. If the old sleep set contained some operations that are not included in the new sleep set, the node is expanded again with a sleep set which is the intersection of the new and the old sleep set. This guarantees that if the node is reached from two or more directions, it will provide enough successors for all of them.

Whereas the sleep sets represents a different approach for global states based model checking, McMillan's unfolding principle [19] is based directly the partial order model of execution. It constructs a structure of partially ordered local states. The order between events represents the causal order on their execution. The unfolding algorithm generates a representation of the checked system which is sometimes called an event structure. It thus avoids generating the global states of the system altogether. The original unfolding algorithm was designed for finding deadlocks. Extensions of this algorithm, e.g., by Esparza [7], were developed for checking different properties.

In cases where partial order methods fail, other techniques for reducing the state space may be more effective. Composing different methods may result in the ability to verify more diverse systems, or even obtaining a more significant reduction than can be achieved by each method separately. However, the combination does not always follow trivially from the joint application of separate techniques. Partial order reduction has been combined with various other model checking methods as follows:

- Partial order can be performed with on-the-fly model checking [17], i.e., generating the reduced state space at the same time as checking for counterexamples for the checked property. The construction can result in such a counterexample before completing the generation of the entire state graph [22, 24].

- Symmetry reduction can be used to obtain a smaller state space when dealing with systems of identical components. Partial order reduction and symmetry were combined in [6].

- Symbolic model checking is a very effective method, which uses the BDD data structure for manipulating and storing the states. Although it is generally used for synchronous hardware systems, where partial order reduction is not effective, symbolic model checking was shown to give very good results for asynchronous systems, including software as well. One way of combining this method with the partial order reduction was suggested in [1], where a reduction based on breadth first search was used [2]. A different way of combining these methods is suggested in [18], based on statically resolving possible cycles of the constructed reduced state space.

Partial order reduction can also be applied to branching temporal logic and process algebra [9], and be used under fairness assumptions [21]. Ongoing research on partial order reduction seeks improved versions of the reduction, applications for additional models, and specification formalisms for which reduction is more effective.

\section{References}

1. R. Alur, R. K. Brayton, T. A. Henzinger, S. Qadeer, and S. K. Rajamani. Partial-order reduction in symbolic state space exploration. In O. Grumberg, editor, Proceedings of the Eighth Workshop on Computer-Aided Verification, volume 1254 of Lecture Notes in Computer Science, pages 340-351, Haifa, Israel, June 1997. SpringerVerlag.

2. C.-T. Chou and D. Peled. Verifying a model-checking algorithm. In Tools and Algorithms for the Construction and Analysis of Systems, volume 1055 of Lecture Notes in Computer Science, pages 241-257, Passau, Germany, 1996. Springer-Verlag.

3. E. Clarke and R. Kurshan, editors. Proceedings of the Second Workshop on Computer-Aided Verification, volume 531 of Lecture Notes in Computer Science, New Brunswick, NJ, USA, June 1990. Springer-Verlag.

4. E. M. Clarke, O. Grumberg, and D. A. Peled. Model Checking. M. I. T. Press. In preparation.

5. C. Courcoubetis, editor. Proceedings of the Fifth Workshop on Computer-Aided Verification, volume 697 of Lecture Notes in Computer Science, Elounda, Greece, June 1993. Springer-Verlag.

6. E. A. Emerson, S. Jha, and D. Peled. Combining partial order and symmetry reduction. In Tools and Algorithms for the Construction and Analysis of Systems, volume 1217 of Lecture Notes in Computer Science, pages 19-34, Enschede, The Netherlands, 1997. Springer-Verlag.

7. J. Esparza. Model checking using net unfoldings. Science of Computer Programming, 23(2-3):151-195, 1994.

8. D. Gabbay, A. Pnueli, S. Shelah, and J. Stavi. On the temporal analysis of fairness. In Proceedings of the rth ACM Symposium on Principles of Programming Languages, pages 163-173, Jan. 1980.

9. R. Gerth, R. Kuiper, D. Peled, and W. Penczek. A partial order approach to branching time logic model checking. In Proceedings of the Third Israel Symposium on the Theory of Computing and Systems, pages 130-140. IEEE Computer Society Press, 1995. To appear in Information and Computation.

10. P. Godefroid. Using partial orders to improve automatic verification methods. In Clarke and Kurshan [3], pages 176-185.

11. P. Godefroid and D. Pirottin. Refining dependencies improves partial-order verification methods. In Courcoubetis [5], pages $438-449$.

12. P. Godefroid and P. Wolper. A partial approach to model checking. In Proceedings of Sixth Annual IEEE Symposium on Logic in Computer Science, pages 406-415, Amsterdam, July 1991. 
13. C. A. R. Hoare. Communicating Sequential Processes. Prentice Hall, 1995.

14. G. J. Holzmann. Design and Validation of Computer Protocols. Prentice Hall, 1991.

15. G. J. Holzmann and D. Peled. An improvement in formal verification. In Formal Description Techniques 1994, pages 197-211, Bern, Switzerland, 1994. Chapman \& Hall.

16. S. Katz and D. Peled. An efficient verification method for parallel and distributed programs. In Workshop on Linear Time, Branching Time and Partial Order in Logics and Models for Concurrency, number 354 in Lecture Notes in Computer Science, pages 489-507, Noordwijkerhout, The Netherlands, 1988. Springer-Verlag.

17. R. P. Kurshan. Computer-Aided Verification of Coordinating Processes: The Automata-Theoretic Approach. Princeton University Press, Princeton, New Jersey, 1994.

18. R. P. Kurshan, V. Levin, M. Minea, D. Peled, and H. Yenigun. Verifying hardware in its software context. In Proceedings of the 1997 IEEE International Conference on Computer-Aided Design, San Jose, CA, USA, Nov. 1997. IEEE Computer Society Press.

19. K. L. McMillan. Using unfolding to avoid the state explosion problem in the verification of asynchronous circuits. In G. v. Bochmann and D. K. Probst, editors, Proceedings of the Fourth Workshop on Computer-Aided Verification, volume 663 of Lecture Notes in Computer Science, pages 164-177, Montreal, Canada, June 1992. Springer-Verlag.

20. W. T. Overman. Verification of Concurrent Systems: Function and Timing. PhD thesis, University of California at Los Angeles, 1981.

21. D. Peled. All from one, one for all: on model checking using representatives. In Courcoubetis [5], pages 409-423.

22. D. Peled. Combining partial order reductions with onthe-fly model-checking. In D. L. Dill, editor, Proceedings of the Sixth Workshop on Computer-Aided Verification, volume 818 of Lecture Notes in Computer Science, pages 377-390, Stanford, CA, USA, June 1994. Springer-Verlag.

23. D. Peled and T. Wilke. Stutter-invariant temporal properties are expressible without the next-time operator. Information Processing Letters, 63(5):243-246, 1997.

24. A. Valmari. A stubborn attack on state explosion. In Clarke and Kurshan [3], pages 156-165. 